\title{
Huntingtin-Interacting Protein 1 Promotes Vpr-Induced G2 Arrest and HIV-1 Infection in Macrophages
}

\author{
Tomoyuki Murakami ${ }^{1,+}+\mathbb{C}$, Ryosuke Matsuura ${ }^{1,2,{ }^{\dagger}}$, Nopporn Chutiwitoonchai ${ }^{1}$, Masami Takei ${ }^{3} \mathbb{C}$ \\ and Yoko Aida ${ }^{1,2, *}$ \\ 1 Viral Infectious Diseases Unit, RIKEN, 2-1 Hirosawa, Wako, Saitama 351-0198, Japan; \\ tmurakam@umich.edu (T.M.); matsuura-ryosuke@g.ecc.u-tokyo.ac.jp (R.M.); nponopr@gmail.com (N.C.) \\ 2 Laboratory of Global Infectious Diseases Control Science, Graduate School of Agricultural and Life Sciences, \\ The University of Tokyo, 1-1-1 Yayoi, Bunkyo-ku, Tokyo 113-8657, Japan \\ 3 Division of Hematology and Rheumatology, Department of Medicine, Nihon University School of Medicine, \\ 30-1 Oyaguchi, Kami-cho, Itabashi, Tokyo 173-8610, Japan; takei.masami@nihon-u.ac.jp \\ * Correspondence: yoko-aida@g.ecc.u-tokyo.ac.jp \\ + These authors contributed equally to this work.
}

Citation: Murakami, T.; Matsuura, R.; Chutiwitoonchai, N.; Takei, M.; Aida, Y. Huntingtin-Interacting Protein 1 Promotes Vpr-Induced G2 Arrest and HIV-1 Infection in

Macrophages. Viruses 2021, 13, 2308. https://doi.org/10.3390/v13112308

Academic Editor: Felipe Diaz-Griffero

Received: 8 October 2021

Accepted: 17 November 2021

Published: 19 November 2021

Publisher's Note: MDPI stays neutral with regard to jurisdictional claims in published maps and institutional affiliations.

Copyright: (c) 2021 by the authors. Licensee MDPI, Basel, Switzerland. This article is an open access article distributed under the terms and conditions of the Creative Commons Attribution (CC BY) license (https:// creativecommons.org/licenses/by/ $4.0 /)$.

\begin{abstract}
Human immunodeficiency virus type 1 (HIV-1) modulates the host cell cycle. The HIV-1 accessory protein Vpr arrests the cell cycle at the G2 phase in dividing cells, and the ability of Vpr to induce G2 arrest is well conserved among primate lentiviruses. Additionally, Vpr-mediated G2 arrest likely correlates with enhanced HIV-1 infection in monocyte-derived macrophages. Here, we screened small-interfering RNA to reveal candidates that suppress Vpr-induced G2 arrest and identified Huntingtin-interacting protein 1 (HIP1) required for efficient G2 arrest. Interestingly, HIP1 was not essential for Vpr-induced DNA double-strand breaks, which are required for activation of the DNA-damage checkpoint and G2 arrest. Furthermore, HIP1 knockdown suppressed HIV-1 infection in monocyte-derived macrophages. This study identifies HIP1 as a factor promoting Vpr-induced G2 arrest and HIV-1 infection in macrophages.
\end{abstract}

Keywords: HIV-1; Vpr; G2 arrest; HIP1; macrophage

\section{Introduction}

Vpr is an accessory gene product of the human immunodeficiency virus type 1 (HIV-1) and a small 15-kDa protein with multiple biological functions, including splicing regulation [1-3], support of virus release [4], nuclear import of the viral preintegration complex in macrophages [5-7], enhanced expression and processing of the envelope glycoprotein in macrophages [8-10], sustaining interleukin 6 expression to enhance HIV-1 replication [11], antagonism of exonuclease 1- and helicase-like transcription factor-mediated restriction in T cells through degradation of these proteins [12-15], regulation of apoptosis in both a positive and negative manner, and the induction of cell cycle arrest at the G2 phase in dividing cells [16-21]. Multiple functions of Vpr are exerted through interactions with various host factors, such as DNA damage-binding protein 1 (DDB1)- and cullin 4 (CUL4)-associated factor 1 (DCAF1), spliceosome-associated protein 145, p300, synthetic lethal of unknown (X) function 4 (SLX4), protein arginine N-methyltransferase 5, importin $\alpha$, mini-chromosome maintenance protein10, and coiled-coil domain-containing-137 (CCDC137) $[2,6,7,13,20,22-27]$. The induction of G2 arrest is likely an important function for efficient viral replication because the ability of Vpr to cause cell cycle blockade is well conserved among primate lentiviruses [28,29]. Additionally, Vpr accelerates acute HIV-1 infection by exploiting proliferating $\mathrm{CD} 4^{+} \mathrm{T}$ cells, including regulatory $\mathrm{CD} 4^{+} \mathrm{T}$ cells, through G2 arrest and apoptosis in vivo [30].

Vpr induces DNA damage and activates ataxia telangiectasia-mutated and Rad3related protein (ATR) to induce G2 arrest [31-33]. Vpr-induced G2 arrest requires the association of Vpr with the CUL4 ubiquitin ligase in association with DDB1 and DCAF1 [24,34-38]. 
In addition, recently, it has been shown that Vpr degrades CCDC137 to induce G2 arrest [22]. Although previous reports indicate that the interaction between CUL4-Vpr and the SLX4 complex is necessary for efficient induction of G2 arrest [25,39], Fregoso et al. [40] demonstrated that Vpr induces G2 arrest independent of SLX4. Thus, the full molecular mechanism(s) underlying Vpr-induced G2 arrest remain unknown.

Macrophages are a cellular target of HIV-1. In HIV-1-infected patients, macrophages reportedly act as a viral reservoir widely distributed throughout multiple tissues under the combination antiretroviral therapy [41,42]. Vpr enhances HIV-1 infection in macrophages through various mechanisms [6-12,43], and a Vpr mutant showing defective induction of a G2 arrest also fails to promote HIV-1 infection in macrophages [43]. Therefore, it is possible that Vpr-mediated G2 arrest is implicated in efficient HIV-1 infection in macrophages, and that understanding of Vpr-specific G2-arrest mechanism(s) could reveal how Vpr facilitates HIV-1 infection in macrophages.

Huntingtin-interacting protein 1 (HIP1) interacts with the protein encoded by the gene mutated in Huntington's disease [44,45], an inherited neurodegenerative disorder caused by expansion of the codon CAG in the Huntingtin gene and resulting in the translation of a polyglutamine tract in the protein. The affinity of the Huntingtin-HIP1 interaction is inversely correlated with the polyglutamine-repeat length $[44,45]$. Additionally, HIP1 is associated not only with Huntingtin's disease but also various cellular processes, including clathrin-mediated endocytosis [46-48], tumorigenesis [49,50], and neuronal cell death $[51,52]$.

In this study, we investigated the novel molecular mechanism of Vpr-mediated G2 arrest by screening candidate(s) directly involved in this process through the use of a smallinterfering (si)RNA mini-library of target genes and CELAVIEW RS100, an imaging-based screening microscope. CELAVIEW RS100 automatically acquires cellular fluorescence images and quantitatively analyzes the morphology and fluorescence signal in a large number of cells. Compared with flow cytometry analysis, CELAVIEW RS100 enables highthroughput analysis of DNA contents in the Hoechst33342-stained nuclei of large numbers of Vpr-expressing cells. As a second screening, we determined whether the candidate(s) were involved in Vpr-induced G2 arrest using flow cytometry analysis. Screening results and a further validation experiment identified HIP1 as a novel host factor involved in Vpr-induced G2 arrest, after which we examined the effect of HIP1 knockdown on HIV-1 infection in macrophages.

\section{Materials and Methods}

\subsection{Cell Culture and Transfection}

HeLa cells and 293T cells were grown in Dulbecco's modified Eagle's medium (GIBCO, Gaithersburg, MD, USA) supplemented with $10 \%$ heat-inactivated fetal bovine serum (FCS; Sigma-Aldrich, St. Louis, MO, USA). Human PBMCs were isolated on a Ficoll (Lymphosepal; Immuno-Biological Laboratories, Minneapolis, MN, USA) gradient from a healthy donor. Monocytes were selected from PBMCs using CD14 MicroBeads (Miltenyi Biotec, Bergisch Gladbach, Germany) and a MACS separation column (Miltenyi Biotec) with a Quandro MACS separation unit (Miltenyi Biotec) according to manufacturer instructions. Monocytes were cultured at the desired density in 6- or 24-well plates and grown in Roswell Park Memorial Institute (RPMI)-1640 medium (Invitrogen, Carlsbad, CA, USA) containing 10\% heat-inactivated FCS (Culture Biosciences, San Francisco, CA, USA), 5\% AB serum (Sigma-Aldrich), and a $10 \mathrm{ng} / \mathrm{mL}$ macrophage colony-stimulating factor (M-CSF; PeproTech, Rocky Hill, NJ, USA) for 1 week until they spontaneously differentiated into mature macrophages.

Plasmid transfection was performed using FuGENE HD (Promega, Madison, WI, USA) or Lipofectamine2000 (Invitrogen). siRNA transfection was performed using Lipofectamine2000 or Lipofectamine RNAi MAX (Invitrogen). siRNA and plasmid co-transfection was performed using Lipofectamine2000. siRNA transfection for macrophages was performed using Lipofectamine RNAi MAX. Macrophages were transfected with $50 \mathrm{nM}$ siRNA 
in Opti-MEM (GIBCO). At 4-h post-transfection, macrophages were washed and cultured in an RPMI-1640 medium containing 10\% heat-inactivated FCS, 5\% AB serum, and $10 \mathrm{ng} / \mathrm{mL}$ $\mathrm{M}$-CSF for $20 \mathrm{~h}$. Cells were subjected to subsequent rounds of transfection, washing, and culturing as described.

\subsection{Plasmid Construction}

The expression vector pME18Neo encoding N-terminal Flag-tagged WT Vpr (pME/FVpr) and pGEX-6P-3 encoding N-terminal GST-tagged Vpr (pGEX-6P-3/GST-Vpr) have been described previously $[53,54]$. The molecular clone vectors pNL4-3-Luc-env $(-)$ and pNL4-3-Luc-env(-)vpr(-) and an expression construct for the vesicular stomatitis virus $G$ protein (VSV-G) (pVSV-G) were kindly gifted by Dr. Ishizaka (Department of Intractable Diseases, National Center for Global Health and Medicine). For construction of the vector $\mathrm{pME/F-Vpr-IRES-ZsGreen1} \mathrm{and} \mathrm{the} \mathrm{control} \mathrm{vector} \mathrm{pME/F-IRES-ZsGreen1,} \mathrm{a} \mathrm{fragment} \mathrm{con-}$ taining an IRES sequence and a ZsGreen1-coding sequence was amplified by a polymerase chain reaction (PCR) using the primers $5^{\prime}$-CCCAAACTTAAGCTTGGTACCGA- $3^{\prime}$ and 5'-TAGCGGCCGCTCAGGGCAAGGCGGAGCCGGAG-3' and pRetroX-IRES-ZsGreen1 (Clontech Laboratories, Mountain View, CA, USA) as a template. The PCR fragment was subcloned into pME/F-Vpr and pME18Neo/Flag at the NotI site.

For construction of the expression vector pCAGGS encoding N-terminal HA-tagged HIP1 (pCAGGS/HA-HIP1), human HIP1 mRNA was amplified by reverse transcription (RT)-PCR from RNA derived from HeLa cells. RT was performed with an oligodT primer, and PCR was performed using the primers $5^{\prime}$-AAAGATATCGGATCGGATG GCCAGCTCCATGAAGCAGGTGCCCAA- $3^{\prime}$ and $5^{\prime}$-AAAGCGGCCGCCTATTCTTTTTCG GTTACCACTTC- $3^{\prime}$. The PCR fragment was subcloned into the pCAGGS/HA vector between the EcoRV and NotI sites. For construction of the pCAGGS/HA-siR-HIP1 vector, the mutant was generated using standard PCR mutagenesis techniques using pCAGGS/HAHIP1 as a template.

\subsection{SiRNA}

siRNAs against 256 genes were prepared as an siRNA mini-library [55], and the identities are shown in Kameoka et al. [55]. siRNAs targeting HIP1 and DCAF1 were designed using BLOCK-iT RNAi Designer (Invitrogen). The siRNA forward sequences targeting HIP1 were $5^{\prime}$-CACAGACCUUCUGGUCUGUUGUCAA- $3^{\prime}$ for siRNA\#1 and $5^{\prime}$ GGAGCUAAUGGUGUGUUCUCAUGAA-3' for siRNA\#2. The siRNA forward sequence targeting DCAF1 was 5'-CCCUGGGUGAUUGGCACCAAUUAUA-3'.

\subsection{Western Blotting}

Cells were lysed for $30 \mathrm{~min}$ on ice in $10 \mathrm{mM}$ Tris- $\mathrm{HCl}(\mathrm{pH} 8.0), 150 \mathrm{mM} \mathrm{NaCl}, 5 \mathrm{mM}$ EDTA, $1 \%$ Triton X-100, and $0.1 \%$ SDS supplemented with a protease-inhibitor cocktail (Roche). Lysates were mixed with an SDS-PAGE sample buffer and boiled for $5 \mathrm{~min}$. Protein concentrations were determined with a BCA protein assay kit (Pierce; Thermo Fisher Scientific, Waltham, MA, USA) using bovine serum albumin as a standard. Equal amounts of total protein were examined by Western blotting using the following antibodies: anti-Flag monoclonal antibody (mAb; M2; Sigma-Aldrich), anti-Flag polyclonal antibody (SigmaAldrich), anti-HA polyclonal antibody (Sigma-Aldrich), anti- $\beta$-actin mAb (Sigma-Aldrich), anti-HA mAb (MBL International, Woburn, MA, USA), anti-HIP1 mAb (Novus Biologicals, Littleton, CO, USA), anti-VprBP polyclonal Ab (Proteintech, Rosemont, IL, USA), horseradish-peroxidase (HRP)-conjugated goat anti-mouse IgG (Amersham Biosciences, Little Chalfont, UK), and HRP-conjugated goat anti-rabbit IgG (Amersham Biosciences). Signals were visualized after treatment with SuperSignal West Pico chemiluminescent substrate (Pierce; Thermo Fisher Scientific). 


\subsection{Immunofluorescent Staining}

HeLa cells on a cover slip were co-transfected with siRNAs and either pME/F-VprIRES-ZsGreen1 or pME/F-IRES-ZsGreen1 and cultured for $48 \mathrm{~h}$. Cells were fixed in 3.6\% formaldehyde in a phosphate-buffered saline (PBS) for $10 \mathrm{~min}$ at room temperature (RT) and permeabilized with PBS containing $0.2 \%$ Triton X-100 for 5 min on ice. After treating with the PBS containing $5 \%$ skim milk for $15 \mathrm{~min}$ at RT, the cells were stained with the anti- $\gamma$ $\mathrm{H} 2 \mathrm{AX}$ mAb (Sigma-Aldrich), followed by Alexa Fluor 594-conjugated secondary antibody (Invitrogen) and $5 \mu \mathrm{g} / \mathrm{mL}$ Hoechst33342 in PBS containing 2.5\% skim milk for $30 \mathrm{~min}$. After rinsing with PBS, the cover slip was mounted on glass slides in the PBS containing $90 \%$ glycerol and analyzed by confocal laser scanning microscopy (Olympus, FV1000D).

\subsection{Analysis of Cell Cycle Profiles by CELAVIEW RS100}

HeLa cells were plated in 24-well polystyrene plates. To analyze the effect of siRNA transfection on Vpr-induced G2 arrest, the cells were co-transfected with siRNAs and either pME/F-Vpr-IRES-ZsGreen1 or pME/F-IRES-ZsGreen1 and cultured for $48 \mathrm{~h}$. To analyze the effect of HIP1 overexpression on the inhibition of Vpr-induced G2 arrest following HIP1 knockdown, the cells were co-transfected with siRNAs, pME/F-Vpr-IRESZsGreen1, and pCAGGS/HA-siR-HIP1 or pCAGGS/HA and cultured for $48 \mathrm{~h}$. These cells were fixed and stained with $3.6 \%$ formaldehyde containing $5 \mu \mathrm{g} / \mathrm{mL}$ Hoechst 33342 for $10 \mathrm{~min}$ at room temperature and then washed 3 times with PBS. For each sample, at least 200 ZsGreen1-positive cells were observed and analyzed using a CELAVIEW microscope (RS100; Olympus, Tokyo, Japan).

\subsection{Analysis of Cell Cycle Profiles by Flow Cytometry}

HeLa cells were co-transfected with siRNAs and either pME/F-Vpr-IRES-ZsGreen1 or pME/F-IRES-ZsGreen 1 as a control and cultured for $48 \mathrm{~h}$. The cells were harvested and fixed with $1 \%$ formaldehyde, followed by $70 \%$ ethanol. Fixed cells were incubated in PBS containing RNase A $(50 \mu \mathrm{g} / \mathrm{mL})$ at $37^{\circ} \mathrm{C}$ for $20 \mathrm{~min}$ and then stained with propidium iodide $(40 \mu \mathrm{g} / \mathrm{mL})$. For each sample, at least 7000 cells were analyzed using a FACS Calibur instrument (Becton Dickinson, Franklin Lakes, NJ, USA) with CELL Quest software (Becton Dickinson). Ratios of the numbers of cells in G1 and G2/M phases (G2/M:G1 ratios) were calculated using ModFit LT software (Verity Software House, Topsham, ME, USA).

\subsection{Immunoprecipitation}

293T cells were co-transfected with the indicated vectors, and at 48-h post-transfection, the cells were lysed with a lysis buffer [ $50 \mathrm{mM}$ Tris- $\mathrm{HCl}$ (pH 7.4), $150 \mathrm{mM} \mathrm{NaCl}$, and $0.5 \%$ NP-40] supplemented with a protease-inhibitor cocktail (Roche, Basel, Switzerland) for $30 \mathrm{~min}$ on ice. The lysates were centrifuged at 15,000 rpm for $5 \mathrm{~min}$, and the supernatants were collected and mixed with Anti-HA agarose beads (Sigma-Aldrich) and incubated at $4{ }^{\circ} \mathrm{C}$ for $18 \mathrm{~h}$ with gentle rotation. The beads were washed 5 times with a lysis buffer, and the bound proteins were eluted using the HA peptide (Sigma-Aldrich). Eluted proteins were fractionated by $6 \%$ and $15 \%$ sodium dodecyl sulfate polyacrylamide gel electrophoresis (SDS-PAGE) and analyzed Western blot.

\subsection{Protein Expression and Purification}

Recombinant glutathione-S-transferase (GST) or GST-Vpr was expressed in Escherichia coli BL21 CodonPlus (DE3)-RIL cells (Stratagene, San Diego, CA, USA). Expression was induced with $1 \mathrm{mM}$ isopropyl $\beta$-D-1-thiogalactopyranoside at $16^{\circ} \mathrm{C}$ for $24 \mathrm{~h}$, followed by lysis with a BugBuster reagent (Novagen; Merck, Kenilworth, NJ, USA) according to manufacturer instructions. The lysate was cleared by centrifugation, and the soluble fraction was mixed with glutathione-Sepharose 4 FastFlow beads (GE Healthcare, Pittsburgh, PA, USA), which were centrifuged and washed with a BugBuster reagent and phosphate-buffered saline (PBS). 
To express and purify HA-HIP1, 293T cells were transfected with pCAGGS/HAHIP1 using a FuGene HD transfection reagent (Promega), and at 48-h post-transfection, the cells were collected and lysed with a wash buffer. The lysates were centrifuged at 15,000 rpm for $5 \mathrm{~min}$, and the supernatants were collected, mixed with Anti-HA agarose beads (Sigma-Aldrich), and incubated at $4{ }^{\circ} \mathrm{C}$ for $1 \mathrm{~h}$ with gentle rotation. The affinity beads were washed with a wash buffer twice, and the HA-HIP1 was eluted using the HA peptide (Sigma-Aldrich).

\subsection{Pull-Down Assay}

Purified HA-HIP1 was incubated with GST or GST-Vpr preadsorbed onto glutathioneSepharose 4 FastFlow beads at $4{ }^{\circ} \mathrm{C}$ for $2 \mathrm{~h}$ in a wash buffer. The beads were then washed with the wash buffer 5 times, and bound proteins were eluted by incubation with a sample buffer for SDS-PAGE at $100{ }^{\circ} \mathrm{C}$ for $5 \mathrm{~min}$. Eluted proteins were fractionated by $6 \%$ SDSPAGE for Western blotting.

\subsection{Viral Stock and Viral Infection of Macrophages}

To generate viral stocks, 293T cells were co-transfected with pNL4-3-Luc-env(-) or pNL4-3-Luc-env(-)vpr(-) and pVSV-G using FuGENE HD (Promega), and the virus was harvested at 48-h post-transfection. HIV-1 titers were measured using an anti-p24 enzymelinked immunosorbent assay kit (Ryukyu Immunology, Okinawa, Japan).

Primary macrophages in 24-well plates were inoculated with VSV-G pseudotyped reporter viruses, NL-Luc-E ${ }^{-} \mathrm{R}^{+}$(VSV-G) or NL-Luc-E ${ }^{-} \mathrm{R}^{-}$(VSV-G); $4 \mathrm{ng}$ of $\mathrm{p} 24$ antigen, cultured for 6 days, harvested, and lysed in a luciferase assay substrate (Promega). Infectivity was determined by the measurement of luciferase activity.

\subsection{Statistical Analysis}

Statistical analyses were performed by Prism 8.0 (GraphPad software, San Diego, CA, USA). For two-group comparisons, a two-tailed Student's $t$-test was used. Data are presented as mean \pm SD and were considered statistically significant when the $p$ value was $<0.05$.

\section{Results}

\subsection{HIP1 Enhances Vpr-Induced G2 Arrest}

To identify new cellular factor(s) involved in the Vpr-induced G2 arrest, we used CELAVIEW RS100 for the initial screening in combination with an siRNA mini-library containing 256 siRNAs [55]. The siRNAs in this mini-library target functional genes that are involved in intracellular signal transduction pathways, intracellular transportation processes, and the cytoskeletal system [55]. CELAVIEW RS100 enables high-throughput analysis of DNA contents via automated image acquisition and data analysis. As shown in Figure 1a, HeLa cells were transfected with a bicistronic vector pME18Neo/Flag-Vprinternal ribosomal entry site (IRES)-ZsGreen1 (pME/F-Vpr-IRES-ZsGreen1) encoding Flag tagged-wild-type (WT) Vpr (F-Vpr) and ZsGreen1 as a marker of Vpr expression, together with the siRNAs. At 48-h post-transfection, the cells were fixed and stained with Hoechst33342 for analysis of DNA contents using CELAVIEW RS100. Among the 256 siRNAs, 36 inhibited Vpr-induced G2 arrest in ZsGreen1+ cells (Figure 1a).

To confirm the screening results, we performed secondary screening to investigate the effect of these siRNAs on the cell cycle progression in HeLa cells using flow cytometry analysis (Figure 1b). All siRNAs that we tested showed an inhibitory effect to Vpr-induced G2 arrest.

To validate whether these target genes truly play a role in G2 arrest, we designed siRNAs targeting different protein-coding sequences and analyzed their effects on the cell cycle profile of Vpr-expressing cells using CELAVIEW RS100. We found that among the siRNAs, only siRNAs targeting HIP1 (\#1 and \#2), which is predominantly expressed in the brain and interacts with Huntingtin [44,45], inhibited Vpr-induced G2 arrest (Figure 2a). 
In addition, these siRNAs did not affect cell cycle progression in cells transfected with pME18Neo/Flag-IRES-ZsGreen1 (pME/F-IRES-ZsGreen1) and either siRNA (Figure 2a).

a

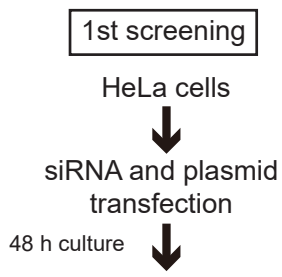

Fixation and staining with Hoechst33342 in 24 well pate without cell harvest

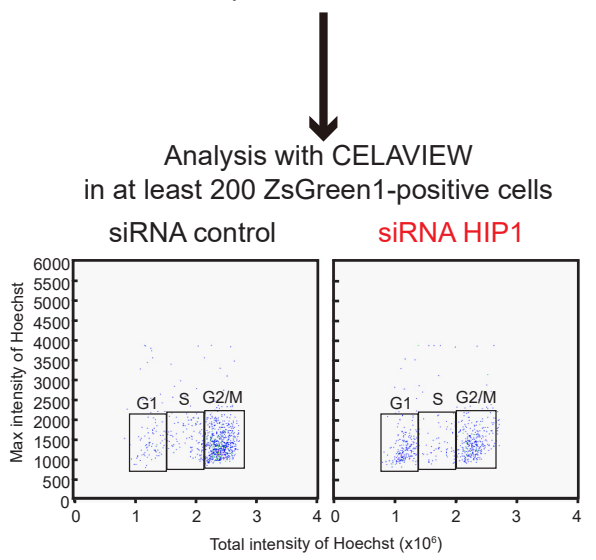

b

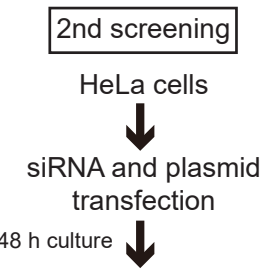

Cell harvest by trypsinization

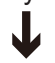

Fixation, permeabilization, and staining with propidium iodide

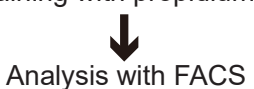

in at least 7000 ZsGreen1-positive cells

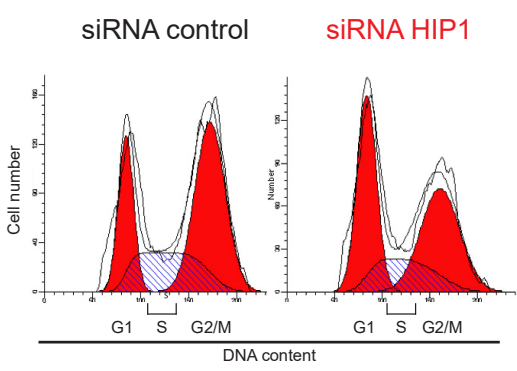

Figure 1. Summary of siRNA screening for candidate suppressors of Vpr-induced G2 arrest. (a) For the initial screening, HeLa cells were co-transfected with pME/F-Vpr-IRES-ZsGreen1 and $100 \mathrm{nM}$ individual siRNAs from the siRNA mini-library or control siRNA. At 48-h post-transfection, cells were fixed and stained with $5 \mu \mathrm{g} / \mathrm{mL}$ Hoechst33342 to measure DNA content in at least 200 ZsGreen1+ cells using CELAVIEW RS100. (b) For secondary screening, HeLa cells were cotransfected with pME/F-Vpr-IRES-ZsGreen1 and control siRNA or $100 \mathrm{nM}$ of individual siRNAs that inhibited Vpr-induced G2 arrest according to the initial screen. At 48-h post-transfection, cells were harvested by trypsinization, fixed, permeabilized, treated with RNase A, and stained with $50 \mu \mathrm{g} / \mathrm{mL}$ propidium iodide to measure DNA content in at least 7000 ZsGreen1+ cells by flow cytometry. The traces on the cell cycle histograms are written by the ModFit LT software automatically.

These results suggest that most siRNAs in the library possibly attenuate Vpr-induced G2 arrest through an off-target effect and that HIP1 could contribute to Vpr-induced G2 arrest. Subsequently, we focused on the role of HIP1 in Vpr-induced G2 arrest. Western blotting confirmed the knockdown of HIP1 levels in HeLa cells transfected with either pME/F-IRES-ZsGreen1 or pME/F-Vpr-IRES-ZsGreen1 together with either newly designed siRNA\#1 or \#2 (Figure 2b). Although transfection with siRNA\#2 reduced the level of Vpr, siRNA\#1 did not affect Vpr expression (Figure 2b). Therefore, these results suggested that since both siRNA\#1 and \#2 similarly inhibited Vpr-induced G2 arrest (Figure 2a), HIP1 knockdown can suppress Vpr-induced G2 arrest without reducing the level of Vpr expression. To examine whether the overexpression of siRNA-resistant HIP1 (siR-HIP1), which is not knocked down by siRNA\#2 due to its synonymous nucleotide mutations in the third codon of the siRNA\#2-targeted sequence, restores the ability of Vpr to induce G2 arrest, HeLa cells were co-transfected with pME/F-Vpr-IRES-ZsGreen1, either siRNA\#2 or control siRNA, and either an siR-HIP1 expression vector or empty vector. We thus observed the ectopic expression of siR-HIP1 even when HeLa cells were co-transfected with siRNA\#2 (Figure 2c), with the overexpression of siR-HIP1 restoring G2 arrest by up to $\sim 40 \%$ (Figure 2d). These results suggested that HIP1 augments Vpr-induced G2 arrest. 

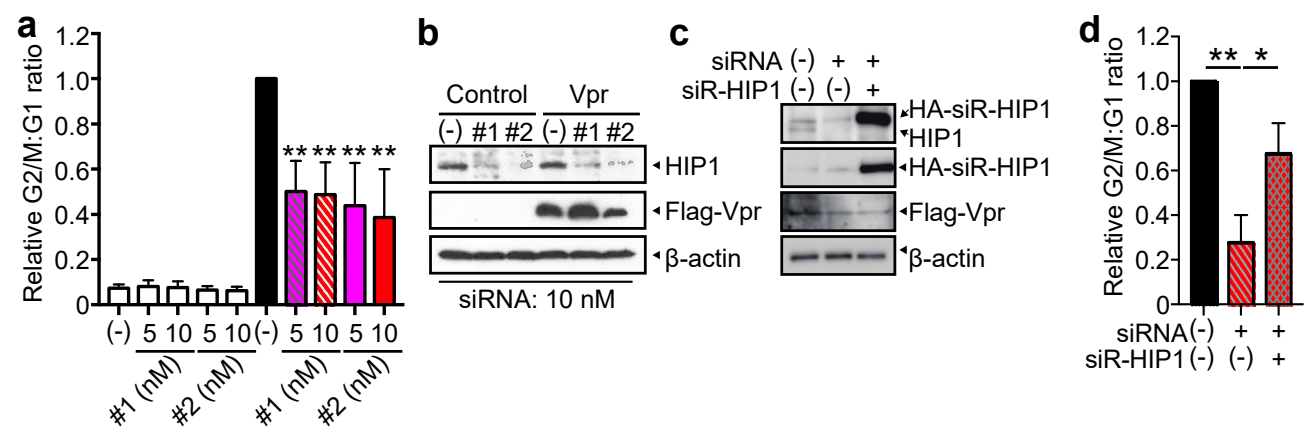

Figure 2. HIP1 enhances Vpr-induced G2 arrest. (a,b) HeLa cells were co-transfected with pME/FVpr-IRES-ZsGreen1 or control pME/F-IRES-ZsGreen1 and 5 or $10 \mathrm{nM}$ either HIP1 (siRNA\#1 and \#2) or control siRNA and cultured for $48 \mathrm{~h}$. (a) Cells were fixed and stained with $5 \mu \mathrm{g} / \mathrm{mL}$ Hoechst33342 to measure DNA content in ZsGreen1+ cells using CELAVIEW RS100. The relative G2/M:G1 ratio was plotted, and data represent the mean \pm SD of three independent experiments. (b) Cells were lysed and subjected to $6 \%$ and 15\% SDS-PAGE and Western blot using an anti-HIP1 mAb, anti-Flag M2 $\mathrm{mAb}$, and anti- $\beta$-actin mAb. (c,d) HeLa cells were co-transfected with pME/F-Vpr-IRES-ZsGreen1 and pCAGGS/HA-siR-HIP1 (carrying synonymous nucleotide mutations at the third codon of the siRNA-targeting site) or control pCAGGS/HA and either 10 nM HIP1 (siRNA\#2) or control siRNA and then cultured for $48 \mathrm{~h}$. (c) Cells were lysed and subjected to 6\% and 15\% SDS-PAGE and Western blot using an anti-HIP1 mAb, anti-Flag M2 mAb, anti-HA mAb, and anti- $\beta$-actin mAb. (d) Cells were fixed, permeabilized, stained with the anti-HA mAb, and then with Alexa Fluor 594-conjugated secondary Ab and Hoechst33342. The DNA content of ZsGreen1+ and Alexa Fluor 594+ cells was analyzed by CELAVIEW RS100. The relative G2/M:G1 ratio was plotted, and data represent the mean \pm SD of three independent experiments. ${ }^{*} p<0.05,{ }^{* *} p<0.01$ via two-tailed Student's $t$-test.

\subsection{HIP1 Interacts with $\mathrm{Vpr}$}

To investigate whether HIP1 interacts with Vpr, we used a GST pull-down assay. Incubating recombinant GST-tagged Vpr (GST-Vpr; immobilized on glutathione-Sepharose beads) with an HA-tagged HIP1 protein (HA-HIP1) purified from 293T cells transfected with pCAGGS/HA-HIP1, followed by GST pull-down assays, revealed that HIP1 bound Vpr (Figure 3a). To confirm the interaction between HIP1 and Vpr in cells, we performed immunoprecipitation assays using lysate from 293T cells transfected with pME18Neo/FlagVpr together with pCAGGS/HA-HIP1, which revealed an HIP1-Vpr interaction (Figure 3b). These results suggested that Vpr possibly induces G2 arrest via interactions with HIP1.
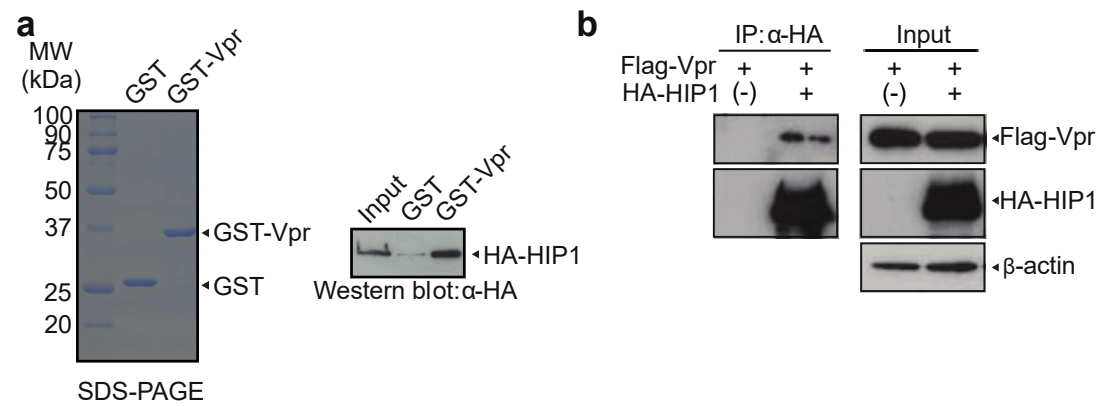

Figure 3. Vpr interacts with HIP1. (a) GST and GST-Vpr were resolved by $12 \%$ SDS-PAGE and stained with Coomassie Brilliant Blue (CBB) (left). Glutathione-Sepharose beads combined with the GST-Vpr or GST alone were incubated with purified HA-HIP1 protein. The bound fractions and $10 \%$ of the input were analyzed by Western blot using the anti-HA mAb (right). The positions of HA-HIP1, GST-Vpr, and GST are indicated. (b) 293T cells were co-transfected with pME/FVpr and pCAGGS/HA-HIP1 or control pCAGGS/HA. At 48-h post-transfection, cells were lysed, and the lysates were subjected to immunoprecipitation assays using anti-HA agarose and the HA peptide. The bound fractions and inputs were analyzed by Western blotting using the anti-HA polyclonal Ab, anti-Flag polyclonal $\mathrm{Ab}$, and anti- $\beta$-actin $\mathrm{mAb}$. 


\subsection{HIP1 Is Dispensable for Vpr-Induced DNA Double-Strand Breaks}

Vpr induces DNA double-strand breaks and G2 arrest via interactions with the CUL4 ubiquitin ligase in association with DDB1 and DCAF1 $[24,34-38,56]$. To test whether HIP1 contributes to Vpr-induced DNA double-strand breaks, we analyzed the effect of HIP1 knockdown on the Vpr-induced formation of DNA-repair foci containing phosphorylated histone 2A variant $X(\gamma-\mathrm{H} 2 \mathrm{AX})$, which forms distinct nuclear foci following Vpr-induced DNA double-strand breaks [32]. After transfection with pME/F-Vpr-IRES-ZsGreen1 together with HIP1 siRNA\#2 and / or DCAF1 siRNA (as a positive control siRNA for inhibition of Vpr-induced DNA double-strand breaks [56]), we performed immunofluorescence staining and confocal microscopy analysis to detect $\gamma$-H2AX foci. Figure 4a shows Western blot confirmation of decreased HIP1 and DCAF1 levels. Interestingly, HIP1 knockdown alone did not suppress Vpr-induced formation of $\gamma$-H2AX foci (Figure $4 b$ ). By contrast, knockdown of DCAF1 alone or both HIP1 and DCAF1 completely inhibited Vpr-induced formation of $\gamma-\mathrm{H} 2 \mathrm{AX}$ foci (Figure $4 \mathrm{~b}$ ). These results indicated that HIP1 is dispensable for Vpr-induced DNA double-strand breaks, and that HIP1 possibly plays a role in Vprinduced G2 arrest independent of DCAF1.

a

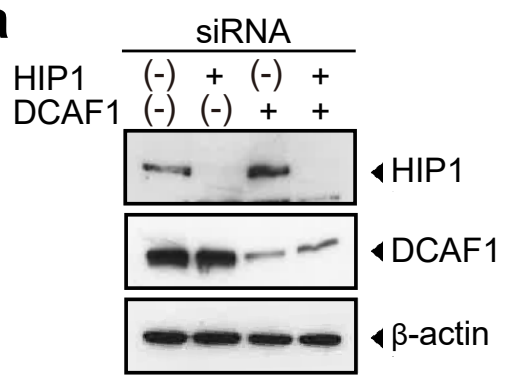

b

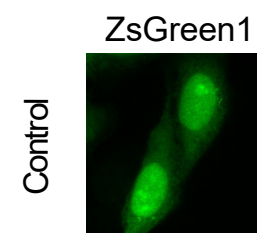

(
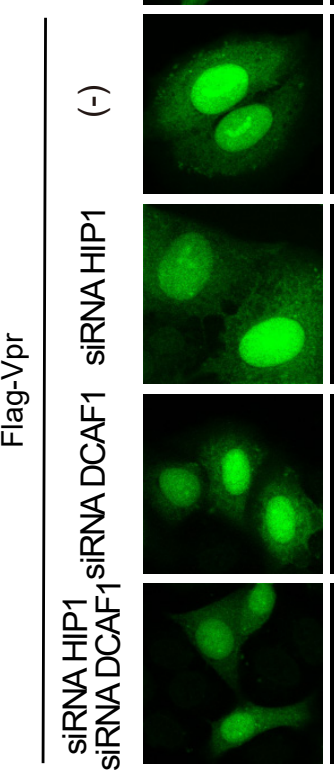
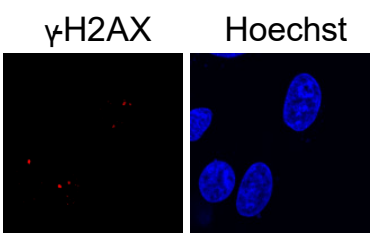

Merge
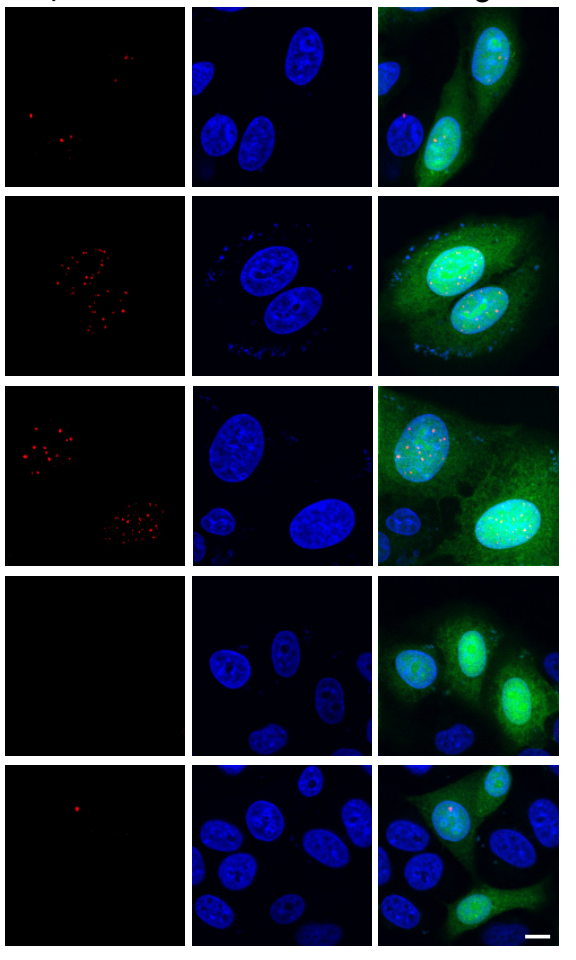

Figure 4. Vpr induces DNA double-strand breaks in HIP1-knockdown cells. (a) HeLa cells were transfected with $5 \mathrm{nM} H I P 1$ (siRNA\#2), DCAF1, or a combination of $5 \mathrm{nM}$ HIP1 (siRNA\#2) and $5 \mathrm{nM}$ DCAF1 siRNAs. The total amount of siRNA was adjusted to $10 \mathrm{nM}$ with control siRNA. At 48-h post-transfection, cells were lysed and subjected to SDS-PAGE and Western blot using the anti-HIP1 mAb, anti-DCAF1 mAb, and anti- $\beta$-actin mAb. The positions of HIP1, DCAF1, and $\beta$-actin are indicated. (b) HeLa cells were co-transfected with pME/F-Vpr-IRES-ZsGreen1 or control pME/F-IRES-ZsGreen1 and $5 \mathrm{nM}$ HIP1 (siRNA\#2), DCAF1, or a combination of $5 \mathrm{nM} \mathrm{HIP1} \mathrm{(siRNA \# 2)} \mathrm{and/or} 5 \mathrm{nM} \mathrm{DCAF1} \mathrm{siRNAs.} \mathrm{The} \mathrm{total} \mathrm{amount} \mathrm{of}$ siRNA was adjusted to $10 \mathrm{nM}$ with control siRNA. At 48-h post-transfection, cells were fixed, permeabilized, and stained with the anti- $\gamma-\mathrm{H} 2 \mathrm{AX} \mathrm{mAb}$, followed by Alexa Fluor 594-conjugated secondary antibody and $5 \mu \mathrm{g} / \mathrm{mL}$ Hoechst33342. Cells were analyzed by a confocal microscopy. Cells showing green fluorescence (ZsGreen1+) and red foci indicated the presence of DNA double-strand breaks, and blue fluorescence indicated nuclei. The scale bar represents $10 \mu \mathrm{m}$. 


\subsection{HIP1 Promotes HIV-1 Infection in Macrophages}

In primary macrophages, Vpr enhances HIV-1 infection, whereas a Vpr mutant that is unable to induce G2 arrest does not enhance HIV-1 infection in macrophages [43]. Therefore, it is possible that HIP1 also contributes to efficient HIV-1 infection in macrophages. To investigate whether HIP1 is involved in enhancing Vpr-mediated HIV-1 infection in primary macrophages, monocyte-derived macrophages were differentiated from monocytes isolated from human peripheral blood mononuclear cells (PBMCs), as described previously $[7,27]$. These macrophages were transfected with HIP1 siRNA\#2 and then infected with VSV-G-pseudotyped NL4-3-Luc HIV-1 encoding either WT Vpr (Vpr+ virus) or truncated $\operatorname{Vpr}\left(\mathrm{Vpr}^{-}\right.$virus), which can only support a single round of HIV-1 replication, at 24-h post-transfection. Following the confirmation of attenuated HIP1 levels by Western blotting (Figure 5a), we determined infectivity by measuring luciferase activity at 6-days post-infection. The luciferase activity in both $\mathrm{Vpr}+$ and $\mathrm{Vpr}^{-}$virus-infected cells was reduced by HIP1 knockdown, with the reduction in Vpr+ virus-infected cells slightly larger than that in $\mathrm{Vpr}^{-}$virus-infected cells ( $\sim 3 \%$ in $\mathrm{Vpr}+$ virus vs. $~ 33 \%$ in $\mathrm{Vpr}^{-}$ virus) (Figure $5 b$ ). These results suggested that although HIP1 contributes to HIV-1 infection in macrophages, it likely promotes stronger infection-related activity via cooperation with Vpr.

a

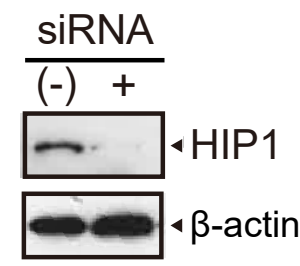

b

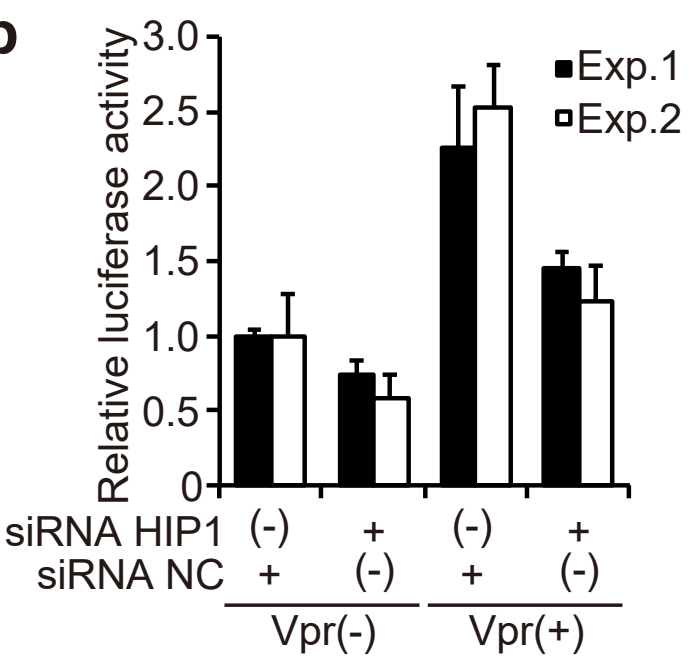

Figure 5. HIP1 enhances HIV-1 infection in macrophages in a Vpr-dependent manner. Monocytes isolated from PBMCs of human healthy donor were subsequently differentiated into macrophages by addition of M-CSF, followed by their transfection with 50 nM HIP1 (siRNA\#2; siRNA HIP1) and control siRNA (siRNA NC). (a) At 48-h post-transfection, cells were lysed and subjected to $6 \%$ SDS-PAGE and Western blotting using the anti-HIP1 mAb and anti- $\beta$-actin mAb. The positions of HIP1 and $\beta$-actin are indicated. (b) At 24-h post-transfection, cells were infected with $4 \mathrm{ng}$ p24 VSV-G-pseudotyped-HIV-1 or -HIV-1 $\Delta$ Vpr. At 6-days post-infection, cells were lysed, and infectivity was determined by measuring luciferase activity. Data represent the mean $\pm \mathrm{SD}$ of triplicate wells.

\section{Discussion}

Vpr induces cell cycle arrest at the G2 phase and promotes HIV-1 infection, especially in macrophages [6-12,18]. The abilities of Vpr to induce G2 arrest and promote HIV-1 infection in macrophages probably correlate with each other. Indeed, Vpr causes G2 arrest and enhances HIV-1 gene expression in both primary $\mathrm{CD}^{+} \mathrm{T}$ cells and macrophages through the depletion of CCDC137 [22], and a Vpr-mutant that is defective in G2 arrest is not able to augment HIV-1 infection in macrophages [43]. Therefore, identifying the host genes with a role in Vpr-induced G2 arrest will possibly lead to an understanding of how Vpr enhances HIV-1 infection in macrophages. Various factors are required for Vpr-induced G2 arrest, including activation of the ATR pathway and interaction with CUL4 ubiquitin ligase in association with DDB1 and DCAF1 [24,25,31-34,36-38,57]. In 
the present study, siRNA screening identified HIP1 as a new host factor that modulates Vpr-induced G2 arrest. We demonstrated that HIP1 enhances Vpr-induced G2 arrest but is not required for Vpr-induced DNA double-strand breaks. Additionally, we found that HIP1 interacts with Vpr. Furthermore, we revealed that HIP1 contributes to efficient HIV-1 infection in macrophages.

Notably, we demonstrated that HIP1 is not necessary for Vpr-induced DNA doublestrand breaks. However, it remains to be determined how HIP1 promotes Vpr-induced G2 arrest, and we thus propose three possibilities as follows: (i) HIP1 plays a role in a DNA damage response signal transduction pathway, (ii) HIP1 contributes to the inhibition of double-strand break repair mediated by Vpr, or (iii) HIP1 attenuates G1 arrest in Vprexpressing cells. As for (i), after Vpr induces DNA double-strand breaks, the DNA damage response is activated to arrest the cell cycle at the G2 phase. The DNA damage response is a signal transduction pathway, which consists of various factors, such as ATR and checkpoint kinases [58]. Therefore, HIP1 might play a role in the signal transduction pathway but not in the induction of DNA damage. Related to this, $\mathrm{Li}$ et al. [59] reported that although protein phosphatase 2 (PP2A) is important for Vpr-induced G2 arrest, Vpr-induced DNA doublestrand breaks are not inhibited by $P P 2 A$ knockdown. Thus, HIP1 possibly collaborates with PP2A to enhance Vpr-induced G2 arrest independent of DNA damage induction. As for (ii), recently, it has been reported that in addition to the induction of DNA damage, Vpr suppresses DNA break repair to arrest the cell cycle at the G2 phase [60]. A mutant Vpr, R80A, which fails to induce G2 arrest, can induce DNA damage but not inhibit DNA repair [60]. Therefore, since HIP1 is dispensable for Vpr-induced double-strand breaks, HIP1 might be involved in the Vpr-mediated inhibition of DNA break repair. As for (iii), even when HIP1 is knocked down, Vpr induces DNA double-strand breaks, suggesting that the DNA damage response is active in Vpr-expressing cells without the expression of HIP1. Thus, the cell cycle of Vpr-expressing cells might be arrested at the G1 phase in the absence of HIP1. Previously, our group reported that a carboxy-terminally truncated mutant of Vpr, C81, induces G1 arrest but not G2 arrest [61]. Therefore, C81 might not be able to cooperate with HIP1 to induce G2 arrest.

We found that HIP1 interacts with Vpr. However, it is not clear whether Vpr requires interaction with HIP1 to induce G2 arrest and facilitate HIV-1 infection in macrophages. If the interaction is essential for efficient Vpr-induced G2 arrest and HIV-1 infection in macrophages, whether Vpr interacts with HIP1 directly or indirectly, whether the interaction between Vpr and HIP1 alters the structure of Vpr, whether HIP1 affects the binding of Vpr to other host proteins via the interaction, and which domains of Vpr and HIP1 mediate the interaction remain to be determined. HIP1 binds to actin through the talin-like domain [62]. Vpr also likely binds to cytoplasmic actin [63]. Therefore, the talin-like domain of HIP1 might be required for interactions with Vpr through actin in the cytoplasm. Even if the interaction between HIP1 and Vpr is not necessary for efficient Vpr-induced G2 arrest and HIV-1 infection in macrophages, the presence of HIP1 might affect the interaction between $\mathrm{Vpr}$ and other host proteins. Therefore, it would be interesting to know whether the knockdown of HIP1 alters interactors of Vpr.

In the present study, we showed that compared to that with Vpr-deleted HIV-1, HIP1 knockdown slightly but effectively inhibited WT HIV-1 infection in macrophages. This result suggested that HIV-1 replication in macrophages is enhanced by HIP1 in corporation with Vpr. In addition to that in macrophages, Vpr enhances HIV-1 infection and HIV-1 gene expression in $\mathrm{CD}^{+} \mathrm{T}$ cells $[15,16,22,64,65]$. Therefore, additional studies are needed to determine whether the knockdown/knockout of HIP1 could affect the Vpr-mediated enhancement of HIV-1 replication in $\mathrm{CD}^{+} \mathrm{T}$ cells.

In HIV-infected $\mathrm{CD} 4^{+} \mathrm{T}$ cells, Vpr induces $\mathrm{G} 2$ arrest [18], suggesting that the knockdown/knockout of HIP1 suppresses Vpr-induced G2 arrest in infected CD4 ${ }^{+} \mathrm{T}$ cells. However, the ability of Vpr to induce G2 arrest is dispensable for the cytopathic effects of Vpr in infected cells [66]. Thus, the knockdown/knockout of HIP1 in CD4 ${ }^{+} \mathrm{T}$ cells would not completely block HIV-1's deleterious effects. 
In summary, the results of the present study suggest that HIP1 augments Vpr-induced G2 arrest and HIV-1 infection in macrophages and that HIP1 interacts with Vpr. However, whether Vpr-induced G2 arrest and Vpr-mediated enhancement of HIV-1 infection requires the interaction between HIP1 and Vpr needs to be clarified. Although HIP1 slightly but efficiently enhanced WT HIV-1 infection in macrophages relative to that by Vpr-deleted HIV-1, the associated mechanism(s) remain to be elucidated. Future investigations are required to determine the exact roles of HIP1 in Vpr-induced G2 arrest and HIV-1 infection in macrophages, as well as to provide insights into correlations between the two functions of $\mathrm{Vpr}$.

Author Contributions: Conceptualization, Y.A. and T.M.; Methodology, Y.A. and T.M.; Validation, Y.A. and T.M.; Formal Analysis, Y.A., T.M. and R.M.; Investigation, T.M., R.M. and N.C.; Resources, Y.A. and M.T. Writing-Original Draft Preparation, Y.A. and T.M.; Writing-Review \& Editing, Y.A. and T.M.; Visualization, T.M.; Supervision, Y.A.; Project Administration, Y.A.; Funding Acquisition, Y.A. All authors have read and agreed to the published version of the manuscript.

Funding: This study was partially supported by a grant (Research on HIV / AIDS project no. H22-003) from the Ministry of Health, Labor, and Welfare of Japan, and by grants (Research on HIV / AIDS. 16fk0410104j0001, and Interdisciplinary Cutting-edge Research projects no JP21wm0325017) from the Japan Agency for Medical Research and Development.

Institutional Review Board Statement: Ethics approval for this study was granted by the RIKEN Ethics Committees [Certificate No. Wako 24-7] and by the Nihon University School of Medicine Ethics Committees [Certificate No. 22-7-10].

Informed Consent Statement: Informed consent was obtained from all subjects involved in the study.

Data Availability Statement: The data presented in this study are available on request from the corresponding author.

Acknowledgments: We thank Naoki Yamamoto of National University of Singapore, Hironori Sato of National Institute of Infectious Diseases, and Jun Komano of Osaka Prefectural Institute of Public Health for kindly providing of siRNA library. We are grateful to the RIKEN BSI-Olympus Collaboration Center for imaging equipment and software, and RIKEN BSI Research Resources Center for help with sequence analysis.

Conflicts of Interest: The authors declare no conflict of interest.

\section{References}

1. Hashizume, C.; Kuramitsu, M.; Zhang, X.; Kurosawa, T.; Kamata, M.; Aida, Y. Human immunodeficiency virus type 1 Vpr interacts with spliceosomal protein SAP145 to mediate cellular pre-mRNA splicing inhibition. Microbes Infect. 2007, 9, 490-497. [CrossRef]

2. Kuramitsu, M.; Hashizume, C.; Yamamoto, N.; Azuma, A.; Kamata, M.; Yamamoto, N.; Tanaka, Y.; Aida, Y. A novel role for Vpr of human immunodeficiency virus type 1 as a regulator of the splicing of cellular pre-mRNA. Microbes Infect. 2005, 7, 1150-1160. [CrossRef] [PubMed]

3. Zhang, X.; Aida, Y. HIV-1 Vpr: A novel role in regulating RNA splicing. Curr. HIV Res. 2009, 7, 163-168. [CrossRef]

4. Chutiwitoonchai, N.; Siarot, L.; Takeda, E.; Shioda, T.; Ueda, M.; Aida, Y. HIV-1 Vpr Abrogates the Effect of TSG101 Overexpression to Support Virus Release. PLoS ONE 2016, 11, e0163100. [CrossRef]

5. Kamata, M.; Nitahara-Kasahara, Y.; Miyamoto, Y.; Yoneda, Y.; Aida, Y. Importin- $\alpha$ Promotes Passage through the Nuclear Pore Complex of Human Immunodeficiency Virus Type 1 Vpr. J. Virol. 2005, 79, 3557-3564. [CrossRef] [PubMed]

6. Nitahara-Kasahara, Y.; Kamata, M.; Yamamoto, T.; Zhang, X.; Miyamoto, Y.; Muneta, K.; Iijima, S.; Yoneda, Y.; Tsunetsugu-Yokota, Y.; Aida, Y. Novel Nuclear Import of Vpr Promoted by Importin $\alpha$ Is Crucial for Human Immunodeficiency Virus Type 1 Replication in Macrophages. J. Virol. 2007, 81, 5284-5293. [CrossRef] [PubMed]

7. Popov, S.; Rexach, M.; Zybarth, G.; Reiling, N.; Lee, M.; Ratner, L.; Lane, C.M.; Moore, M.S.; Blobel, G.; Bukrinsky, M. Viral protein $\mathrm{R}$ regulates nuclear import of the HIV-1 pre-integration complex. EMBO J. 1998, 17, 909-917. [CrossRef]

8. Mashiba, M.; Collins, D.R.; Terry, V.H.; Collins, K.L. Vpr Overcomes Macrophage-Specific Restriction of HIV-1 Env Expression and Virion Production. Cell Host Microbe 2014, 16, 722-735. [CrossRef]

9. Zhang, X.; Zhou, T.; Frabutt, D.; Zheng, Y.-H. HIV-1 Vpr increases Env expression by preventing Env from endoplasmic reticulum-associated protein degradation (ERAD). Virology 2016, 496, 194-202. [CrossRef]

10. Wang, Q.; Su, L. Vpr Enhances HIV-1 Env Processing and Virion Infectivity in Macrophages by Modulating TET2-Dependent IFITM3 Expression. mBio 2019, 10, e01344-19. [CrossRef] 
11. Lv, L.; Wang, Q.; Xu, Y.; Tsao, L.-C.; Nakagawa, T.; Guo, H.; Su, L.; Xiong, Y. Vpr Targets TET2 for Degradation by CRL4VprBP E3 Ligase to Sustain IL-6 Expression and Enhance HIV-1 Replication. Mol. Cell 2018, 70, 961-970.e5. [CrossRef]

12. Hrecka, K.; Hao, C.; Shun, M.-C.; Kaur, S.; Swanson, S.K.; Florens, L.; Washburn, M.; Skowronski, J. HIV-1 and HIV-2 exhibit divergent interactions with HLTF and UNG2 DNA repair proteins. Proc. Natl. Acad. Sci. USA 2016, 113, E3921-E3930. [CrossRef]

13. Lahouassa, H.; Blondot, M.-L.; Chauveau, L.; Chougui, G.; Morel, M.; Leduc, M.; Guillonneau, F.; Ramirez, B.C.; Schwartz, O.; Margottin-Goguet, F. HIV-1 Vpr degrades the HLTF DNA translocase in T cells and macrophages. Proc. Natl. Acad. Sci. USA 2016, 113, 5311-5316. [CrossRef] [PubMed]

14. Yan, J.; Shun, M.-C.; Hao, C.; Zhang, Y.; Qian, J.; Hrecka, K.; DeLucia, M.; Monnie, C.; Ahn, J.; Skowronski, J. HIV-1 Vpr Reprograms CLR4 DCAF1 E3 Ubiquitin Ligase to Antagonize Exonuclease 1-Mediated Restriction of HIV-1 Infection. mBio 2018, 9, e01732-18. [CrossRef] [PubMed]

15. Yan, J.; Shun, M.-C.; Zhang, Y.; Hao, C.; Skowronski, J. HIV-1 Vpr counteracts HLTF-mediated restriction of HIV-1 infection in T cells. Proc. Natl. Acad. Sci. USA 2019, 116, 9568-9577. [CrossRef] [PubMed]

16. Murakami, T.; Aida, Y. Visualizing Vpr-Induced G2 Arrest and Apoptosis. PLoS ONE 2014, 9, e86840. [CrossRef] [PubMed]

17. Ayyavoo, V.; Mahboubi, A.; Mahalingam, S.; Ramalingam, R.; Kudchodkar, S.; Williams, W.V.; Green, D.R.; Weiner, D.B. HIV-1 Vpr suppresses immune activation and apoptosis through regulation of nuclear factor kappa B. Nat. Med. 1997, 3, 1117-1123. [CrossRef] [PubMed]

18. Jowett, J.B.; Planelles, V.; Poon, B.; Shah, N.P.; Chen, M.L.; Chen, I.S. The human immunodeficiency virus type 1 vpr gene arrests infected T cells in the G2+ M phase of the cell cycle. J. Virol. 1995, 69, 6304-6313. [CrossRef] [PubMed]

19. Nishizawa, M.; Kamata, M.; Mojin, T.; Nakai, Y.; Aida, Y. Induction of apoptosis by the Vpr protein of human immunodeficiency virus type 1 occurs independently of G(2) arrest of the cell cycle. Virology 2000, 276, 16-26. [CrossRef] [PubMed]

20. Nonaka, M.; Hashimoto, Y.; Takeshima, S.-N.; Aida, Y. The human immunodeficiency virus type 1 Vpr protein and its carboxyterminally truncated form induce apoptosis in tumor cells. Cancer Cell Int. 2009, 9, 20. [CrossRef] [PubMed]

21. Rogel, M.E.; Wu, L.I.; Emerman, M.L. The human immunodeficiency virus type $1 \mathrm{vpr}$ gene prevents cell proliferation during chronic infection. J. Virol. 1995, 69, 882-888. [CrossRef]

22. Zhang, F.; Bieniasz, P.D. HIV-1 Vpr induces cell cycle arrest and enhances viral gene expression by depleting CCDC137. eLife 2020, 9, e55806. [CrossRef]

23. Chang, H.; Siarot, L.; Matsuura, R.; Lo, C.-W.; Sato, H.; Otsuki, H.; Aida, Y. Distinct MCM10 Proteasomal Degradation Profiles by Primate Lentiviruses Vpr Proteins. Viruses 2020, 12, 98. [CrossRef] [PubMed]

24. Hrecka, K.; Gierszewska, M.; Srivastava, S.; Kozaczkiewicz, L.; Swanson, S.K.; Florens, L.; Washburn, M.; Skowronski, J. Lentiviral Vpr usurps Cul4-DDB1[VprBP] E3 ubiquitin ligase to modulate cell cycle. Proc. Natl. Acad. Sci. USA 2007, 104, 11778-11783. [CrossRef] [PubMed]

25. Laguette, N.; Brégnard, C.; Hue, P.; Basbous, J.; Yatim, A.; Larroque, M.; Kirchhoff, F.; Constantinou, A.; Sobhian, B.; Benkirane, M. Premature Activation of the SLX4 Complex by Vpr Promotes G2/M Arrest and Escape from Innate Immune Sensing. Cell 2014, 156, 134-145. [CrossRef]

26. Miyatake, H.; Sanjoh, A.; Murakami, T.; Murakami, H.; Matsuda, G.; Hagiwara, K.; Yokoyama, M.; Sato, H.; Miyamoto, Y.; Dohmae, N.; et al. Molecular Mechanism of HIV-1 Vpr for Binding to Importin- $\alpha$. J. Mol. Biol. 2016, 428, 2744-2757. [CrossRef]

27. Murakami, H.; Suzuki, T.; Tsuchiya, K.; Gatanaga, H.; Taura, M.; Kudo, E.; Okada, S.; Takei, M.; Kuroda, K.; Yamamoto, T.; et al. Protein Arginine N-methyltransferases 5 and 7 Promote HIV-1 Production. Viruses 2020, 12, 355. [CrossRef]

28. Planelles, V.; Jowett, J.B.; Li, Q.X.; Hahn, B.; Chen, I.S. Vpr-induced cell cycle arrest is conserved among primate lentiviruses. J. Virol. 1996, 70, 2516-2524. [CrossRef] [PubMed]

29. Stivahtis, G.L.; Soares, M.A.; Vodicka, M.A.; Hahn, B.H.; Emerman, M. Conservation and host specificity of Vpr-mediated cell cycle arrest suggest a fundamental role in primate lentivirus evolution and biology. J. Virol. 1997, 71, 4331-4338. [CrossRef]

30. Sato, K.; Misawa, N.; Iwami, S.; Satou, Y.; Matsuoka, M.; Ishizaka, Y.; Ito, M.; Aihara, K.; An, D.S.; Koyanagi, Y. HIV-1 Vpr Accelerates Viral Replication during Acute Infection by Exploitation of Proliferating CD4 ${ }^{+}$T Cells In Vivo. PLoS Pathog. 2013, 9, e1003812. [CrossRef]

31. Poon, B.; Jowett, J.B.; Stewart, S.A.; Armstrong, R.W.; Rishton, G.M.; Chen, I.S. Human immunodeficiency virus type 1 vpr gene induces phenotypic effects similar to those of the DNA alkylating agent, nitrogen mustard. J. Virol. 1997, 71, 3961-3971. [CrossRef] [PubMed]

32. Zimmerman, E.S.; Chen, J.; Andersen, J.L.; Ardon, O.; DeHart, J.L.; Blackett, J.; Choudhary, S.K.; Camerini, D.; Nghiem, P.; Planelles, V. Human immunodeficiency virus type 1 Vpr-mediated G2 arrest requires Rad17 and Hus1 and induces nuclear BRCA1 and gamma-H2AX focus formation. Mol. Cell. Biol. 2004, 24, 9286-9294. [CrossRef] [PubMed]

33. Zimmerman, E.S.; Sherman, M.P.; Blackett, J.L.; Neidleman, J.A.; Kreis, C.; Mundt, P.; Williams, S.A.; Warmerdam, M.; Kahn, J.; Hecht, F.M.; et al. Human Immunodeficiency Virus Type 1 Vpr Induces DNA Replication Stress In Vitro and In Vivo. J. Virol. 2006, 80, 10407-10418. [CrossRef] [PubMed]

34. Belzile, J.-P.; Duisit, G.; Rougeau, N.; Mercier, J.; Finzi, A.; Cohen, É.A. HIV-1 Vpr-Mediated G2 Arrest Involves the DDB1CUL4AVPRBP E3 Ubiquitin Ligase. PLoS Pathog. 2007, 3, e85. [CrossRef]

35. DeHart, J.L.; Zimmerman, E.S.; Ardon, O.; Monteiro-Filho, C.M.; Argañaraz, E.R.; Planelles, V. HIV-1 Vpr activates the G2 checkpoint through manipulation of the ubiquitin proteasome system. Virol. J. 2007, 4, 57. [CrossRef] 
36. Le Rouzic, E.; Belaïdouni, N.; Estrabaud, E.; Morel, M.; Rain, J.-C.; Transy, C.; Margottin-Goguet, F. HIV1 Vpr Arrests the Cell Cycle by Recruiting DCAF1/VprBP, a Receptor of the Cul4-DDB1 Ubiquitin Ligase. Cell Cycle 2007, 6, 182-188. [CrossRef]

37. Schröfelbauer, B.; Hakata, Y.; Landau, N.R. HIV-1 Vpr function is mediated by interaction with the damage-specific DNA-binding protein DDB1. Proc. Natl. Acad. Sci. USA 2007, 104, 4130-4135. [CrossRef]

38. Wen, X.; Duus, K.M.; Friedrich, T.D.; de Noronha, C.M.C. The HIV1 Protein Vpr Acts to Promote G2 Cell Cycle Arrest by Engaging a DDB1 and Cullin4A-containing Ubiquitin Ligase Complex Using VprBP/DCAF1 as an Adaptor. J. Biol. Chem. 2007, 282, 27046-27057. [CrossRef]

39. Berger, G.; Lawrence, M.; Hue, S.; Neil, S.J.D. G2/M Cell Cycle Arrest Correlates with Primate Lentiviral Vpr Interaction with the SLX4 Complex. J. Virol. 2014, 89, 230-240. [CrossRef]

40. Fregoso, O.I.; Emerman, M. Activation of the DNA Damage Response Is a Conserved Function of HIV-1 and HIV-2 Vpr That Is Independent of SLX4 Recruitment. mBio 2016, 7, e01433-16. [CrossRef]

41. Orenstein, J.M.; Fox, C.; Wahl, S.M. Macrophages as a Source of HIV During Opportunistic Infections. Science 1997, $276,1857-1861$. [CrossRef]

42. Sattentau, Q.J.; Stevenson, M. Macrophages and HIV-1: An Unhealthy Constellation. Cell Host Microbe 2016, 19, 304-310. [CrossRef] [PubMed]

43. Subbramanian, R.A.; Kessous-Elbaz, A.; Lodge, R.; Forget, J.; Yao, X.-J.; Bergeron, D.; Cohen, E.A. Human Immunodeficiency Virus Type 1 Vpr Is a Positive Regulator of Viral Transcription and Infectivity in Primary Human Macrophages. J. Exp. Med. 1998, 187, 1103-1111. [CrossRef]

44. Kalchman, M.A.; Koide, H.B.; McCutcheon, K.; Graham, R.K.; Nichol, K.; Nishiyama, K.; Kazemi-Esfarjani, P.; Lynn, F.C.; Wellington, C.; Metzler, M.; et al. HIP1, a human homologue of S. cerevisiae Sla2p, interacts with membrane-associated huntingtin in the brain. Nat. Genet. 1997, 16, 44-53. [CrossRef] [PubMed]

45. Wedemeyer, N.; Peoples, R.; Himmelbauer, H.; Wanker, E.E.; Lehrach, H.; Francke, U. Localization of the HumanHIP1Gene Close to the Elastin (ELN) Locus on 7q11.23. Genomics 1997, 46, 313-315. [CrossRef] [PubMed]

46. Metzler, M.; Legendre-Guillemin, V.; Gan, L.; Chopra, V.; Kwok, A.; McPherson, P.S.; Hayden, M. HIP1 Functions in Clathrinmediated Endocytosis through Binding to Clathrin and Adaptor Protein 2. J. Biol. Chem. 2001, 276, 39271-39276. [CrossRef]

47. Mishra, S.K.; Agostinelli, N.R.; Brett, T.J.; Mizukami, I.; Ross, T.; Traub, L. Clathrin- and AP-2-binding Sites in HIP1 Uncover a General Assembly Role for Endocytic Accessory Proteins. J. Biol. Chem. 2001, 276, 46230-46236. [CrossRef] [PubMed]

48. Waelter, S.; Scherzinger, E.; Hasenbank, R.; Nordhoff, E.; Lurz, R.; Goehler, H.; Gauss, C.; Sathasivam, K.; Bates, G.P.; Lehrach, H.; et al. The huntingtin interacting protein HIP1 is a clathrin and alpha-adaptin-binding protein involved in receptor-mediated endocytosis. Hum. Mol. Genet. 2001, 10, 1807-1817. [CrossRef]

49. Rao, D.S.; Bradley, S.; Kumar, P.D.; Hyun, T.; Saint-Dic, D.; Oravecz-Wilson, K.; Kleer, C.G.; Ross, T.S. Altered receptor trafficking in Huntingtin Interacting Protein 1-transformed cells. Cancer Cell 2003, 3, 471-482. [CrossRef]

50. Rao, D.S.; Hyun, T.S.; Kumar, P.D.; Mizukami, I.F.; Rubin, M.A.; Lucas, P.C.; Sanda, M.G.; Ross, T.S. Huntingtin-interacting protein 1 is overexpressed in prostate and colon cancer and is critical for cellular survival. J. Clin. Investig. 2002, 110, 351-360. [CrossRef]

51. Choi, S.A.; Kim, S.J.; Chung, K.C. Huntingtin-interacting protein 1-mediated neuronal cell death occurs through intrinsic apoptotic pathways and mitochondrial alterations. FEBS Lett. 2006, 580, 5275-5282. [CrossRef] [PubMed]

52. Kang, J.E.; Choi, S.A.; Park, J.B.; Chung, K.C. Regulation of the proapoptotic activity of huntingtin interacting protein 1 by Dyrk1 and caspase-3 in hippocampal neuroprogenitor cells. J. Neurosci. Res. 2005, 81, 62-72. [CrossRef] [PubMed]

53. Nishino, Y.; Myojin, T.; Kamata, M.; Aida, Y. Human Immunodeficiency Virus Type 1VprGene Product Prevents Cell Proliferation on Mouse NIH3T3 Cells without the G2Arrest of the Cell Cycle. Biochem. Biophys. Res. Commun. 1997, 232, 550-554. [CrossRef]

54. Takeda, E.; Murakami, T.; Matsuda, G.; Murakami, H.; Zako, T.; Maeda, M.; Aida, Y. Nuclear Exportin Receptor CAS Regulates the NPI-1-Mediated Nuclear Import of HIV-1 Vpr. PLoS ONE 2011, 6, e27815. [CrossRef]

55. Kameoka, M.; Kitagawa, Y.; Utachee, P.; Jinnopat, P.; Dhepakson, P.; Isarangkura-Na-Ayuthaya, P.; Tokunaga, K.; Sato, H.; Komano, J.; Yamamoto, N.; et al. Identification of the suppressive factors for human immunodeficiency virus type-1 replication using the siRNA mini-library directed against host cellular genes. Biochem. Biophys. Res. Commun. 2007, 359, 729-734. [CrossRef] [PubMed]

56. Belzile, J.-P.; Abrahamyan, L.G.; Gerard, F.C.A.; Rougeau, N.; Cohen, É.A. Formation of Mobile Chromatin-Associated Nuclear Foci Containing HIV-1 Vpr and VPRBP Is Critical for the Induction of G2 Cell Cycle Arrest. PLoS Pathog. 2010, 6, e1001080. [CrossRef]

57. Belzile, J.-P.; Richard, J.; Rougeau, N.; Xiao, Y.; Cohen, E.A. HIV-1 Vpr Induces the K48-Linked Polyubiquitination and Proteasomal Degradation of Target Cellular Proteins To Activate ATR and Promote G 2 Arrest. J. Virol. 2010, 84, 3320-3330. [CrossRef]

58. Zhou, B.B.; Elledge, S.J. The DNA damage response: Putting checkpoints in perspective. Nat. Cell Biol. 2000, 408, 433-439. [CrossRef] [PubMed]

59. Li, G.; Elder, R.T.; Qin, K.; Park, H.U.; Liang, D.; Zhao, R.Y. Phosphatase Type 2A-dependent and -independent Pathways for ATR Phosphorylation of Chk1. J. Biol. Chem. 2007, 282, 7287-7298. [CrossRef]

60. Li, D.; Lopez, A.; Sandoval, C.; Doyle, R.N.; Fregoso, O.I. HIV Vpr Modulates the Host DNA Damage Response at Two Independent Steps to Damage DNA and Repress Double-Strand DNA Break Repair. mBio 2020, 11, e00940-20. [CrossRef]

61. Nishizawa, M.; Kamata, M.; Katsumata, R.; Aida, Y. A Carboxy-Terminally Truncated Form of the Human Immunodeficiency Virus Type 1 Vpr Protein Induces Apoptosis via G 1 Cell Cycle Arrest. J. Virol. 2000, 6058-6067. [CrossRef] [PubMed] 
62. Senetar, M.A.; Foster, S.J.; McCann, R.O. Intrasteric Inhibition Mediates the Interaction of the I/LWEQ Module Proteins Talin1, Talin2, Hip1, and Hip12 with Actin. Biochemistry 2004, 43, 15418-15428. [CrossRef]

63. Jäger, S.; Cimermancic, P.; Gulbahce, N.; Johnson, J.R.; McGovern, K.E.; Clarke, S.C.; Shales, M.; Mercenne, G.; Pache, L.; Li, K.; et al. Global landscape of HIV-human protein complexes. Nature 2011, 481, 365-370. [CrossRef] [PubMed]

64. Goh, W.C.; Rogel, M.E.; Kinsey, C.M.; Michael, S.F.; Fultz, P.N.; Nowak, M.A.; Hahn, B.; Emerman, M. HIV-1 Vpr increases viral expression by manipulation of the cell cycle: A mechanism for selection of Vpr in vivo. Nat. Med. 1998, 4, 65-71. [CrossRef]

65. Vermeire, J.; Roesch, F.; Sauter, D.; Rua, R.; Hotter, D.; Van Nuffel, A.; Vanderstraeten, H.; Naessens, E.; Iannucci, V.; Landi, A.; et al. HIV Triggers a cGAS-Dependent, Vpu- and Vpr-Regulated Type I Interferon Response in CD4 ${ }^{+}$T Cells. Cell Rep. 2016, 17, 413-424. [CrossRef] [PubMed]

66. Bolton, D.L.; Lenardo, M.J. Vpr Cytopathicity Independent of $\mathrm{G}_{2} / \mathrm{M}$ Cell Cycle Arrest in Human Immunodeficiency Virus Type 1-Infected CD4 ${ }^{+}$T Cells. J. Virol. 2007, 81, 8878-8890. [CrossRef] 